\title{
Proton-tetraneutron elastic scattering
}

\author{
B. M. Sherrill and C. A. Bertulani* \\ National Superconducting Cyclotron Laboratory, and Department of Physics and Astronomy, Michigan State University, \\ East Lansing, Michigan 48824-1321, USA \\ (Received 30 October 2003; published 24 February 2004)
}

\begin{abstract}
We analyze the elastic scattering of protons on a ${ }^{4} n$ system. This was used as part of the detection technique of a recent experiment [F. M. Marqués et al. Phys. Rev. C 65, 044006 (2002)] to search for the ${ }^{4} n$ (tetraneutron) as a bound particle. We show that it is unlikely that this process alone could yield the events reported in F. M. Marqués et al., [Phys. Rev. C 65, 044006 (2002)], unless the ${ }^{4} n$ has an anomalously large backward elastic scattering amplitude.
\end{abstract}

DOI: 10.1103/PhysRevC.69.027601

PACS number(s): 25.60.Bx, 25.10.+s

The possible existence of the tetraneutron has been discussed theoretically by numerous authors already in the 1960s (see Refs. [1-3] and references therein). Numerous experiments have been performed with negative results ([1-3] and references therein). However, in a recent experiment [1], the existence of bound neutron clusters was studied by fragmentation of intermediate energy (35 MeV/nucleon) ${ }^{14} \mathrm{Be}$ nuclei. As described in Ref. [1], the data analysis was confined (for technical reasons related to the detector response) to 11-18 MeV/nucleon. The fragmentation channel ${ }^{10} \mathrm{Be}+{ }^{4} n$ was observed and the ${ }^{4} n$ system was tentatively described as a bound tetraneutron system.

Theoretical attempts have failed to find the mechanism for the ${ }^{4} n$ binding. Reference [2] concluded that a strong fournucleon force is needed to bind the tetraneutron. This force would unreasonably bind ${ }^{4} \mathrm{He}$ by about $100 \mathrm{MeV}$. Reference [3] showed that a model of the tetraneutron based on two dineutron molecules is also not likely to yield a bound system. Further, Ref. [4] showed that if the ${ }^{4} n$ would form a bound system it would indeed look like two widely separated dineutrons, with rms radii between $7.3 \mathrm{fm}$ and $10.3 \mathrm{fm}$. However, these conclusions were based on a significant change of presently adopted $N N$ potentials.

In this brief report we point out a puzzle in the experimental measurement [1]. We calculate the elastic protontetraneutron cross section and show that it is surprising that the experimental technique used in Ref. [1] could identify a bound ${ }^{4} n$ based on this reaction. In the original experimental paper the ${ }^{4} n$ was identified by the recoil imparted on a proton target. In particular ${ }^{4} n$ was identified by an anomalous large backward recoil of the proton. We claim that, due to the loosely bound character of the tetraneutron (if it existed) this anomalously large proton recoil is unlikely, as the elastic cross section for the proton-tetraneutron system drops many orders of magnitude for large scattering angles. If the data of Ref. [1] are correct, they indicate an anomalously large backward angle cross section or that other processes are contributing.

To calculate the proton- ${ }^{4} n$ scattering cross section one

\footnotetext{
*Electronic address: sherrill@nscl.msu.edu, bertulani@nscl.msu.edu
}

needs the $p-{ }^{4} n$ phase shifts $\delta_{l \pm}$, or an optical potential, both of which are of course unknown. One can construct one theoretically using several recipes. For simplicity we will use here the M3Y interaction [5] to construct $U_{\text {real }}(r)$. The imaginary part of the potential is taken as $U_{\text {imag }}(r)=\lambda U_{\text {real }}(r)$. We adopt a very conservative value, $\lambda=1$, for simplicity [6]. The input in this calculation is the tetraneutron density distribution $\rho(r)$ which is of course unknown. We assume that if the tetraneutron exists it has a large rms radius, due to its loosely-bound character. We take a one-body density distribution with rms radii of $7 \mathrm{fm}$ and $10 \mathrm{fm}$, respectively [7]. This simulates the small binding energy of the system.

The result of the calculation is presented in Fig. 1, for $E_{p}=14 \mathrm{MeV}$ and a ${ }^{4} n$-system with rms radii equal to $7 \mathrm{fm}$ (dashed line) and $10 \mathrm{fm}$ (solid line), respectively.

In Fig. 2 we show the kinematics of the ${ }^{4} n$-proton scattering in the laboratory. In particular, we show the proton energy as a function of its backscattering angle in degrees, in the kinematical allowed region. In the laboratory, the protons would have recoil energies in the interval $E_{p}=21-38 \mathrm{MeV}$, for scattering angles between $95^{\circ}$ and $180^{\circ}$. This is shown by the solid line in Fig. 2. The dashed line is the respective ${ }^{4} n$ energy.

For proton recoil energies in the interval $E_{p} / E_{n}=$ 1.4-2.6 we get a total elastic cross section (from Fig. 1) of about $3.5 \mu \mathrm{b}$, assuming a ${ }^{4} n$ size of $7 \mathrm{fm}\left(\mathrm{a}^{4} n\right.$ size of $10 \mathrm{fm}$ yields a ten times smaller value).

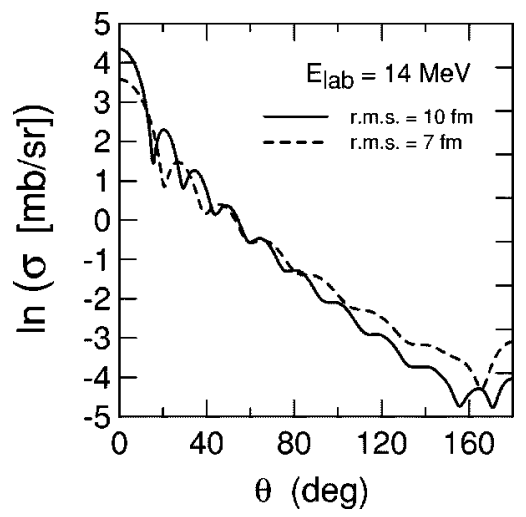

FIG. 1. Proton- ${ }^{4} n$ elastic scattering at $E_{p}=14 \mathrm{MeV}$. The dashed (solid) line assumes a ${ }^{4} n$ matter density distribution with a rms radius of $7 \mathrm{fm}(10 \mathrm{fm})$. 


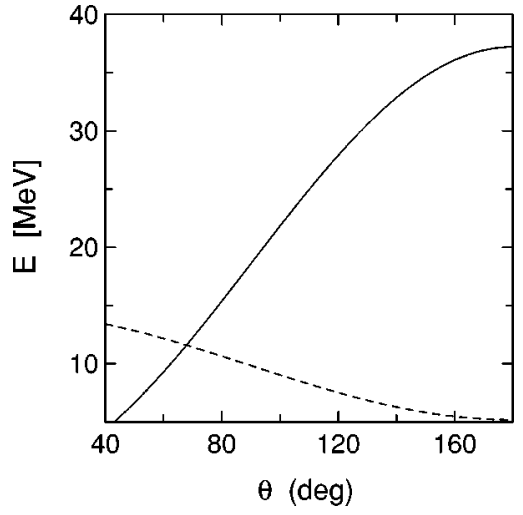

FIG. 2. Kinematics of the ${ }^{4} n$-proton scattering in the laboratory. The solid line shows the proton recoil energies in the interval $E_{p}$ $=21-38 \mathrm{MeV}$, for scattering angles between $95^{\circ}$ and $180^{\circ}$. The dashed line is the respective ${ }^{4} n$ energy (per nucleon).

The intensity of ${ }^{14} \mathrm{Be}$ in the experiment of Ref. [1] is 130 particles per second. Assuming a ${ }^{4} n$ production cross section of $1 \mathrm{mb}$, as estimated in Ref. [1], as an upper estimate and a carbon target with $275 \mathrm{mg} / \mathrm{cm}^{2}$, we obtain $1.8 \times 10^{-2}{ }^{4} \mathrm{n} / \mathrm{s}$. The density of protons in the target can be estimated as $n$ $=10^{24} / \mathrm{cm}^{2}$. For a cross section of $3.5 \mu \mathrm{b}$ one thus gets a counting rate of $10^{-9}$ scattered protons per second. The overall (geometry + response of the detectors) detector efficiency in the experiment is of the order of $10 \%$. This yields approximately $10^{-4}$ events per week. The experiment of Ref. [1] claims six events in a one week run. There is a factor of $10^{4}$ more events than expected from an optimistic estimate.

The estimates presented above show that the results of the experiment cannot be explained as arising from elastic scattering unless there is an anomalous large backscattering in the ${ }^{4} n+p$ system. We think that the ${ }^{4} n$ model used in our calculations should yield an order of magnitude estimate of the distribution of the backscattered protons. It is well known that the elastic scattering cross section decreases exponentially with the diffuseness of the system. For a system of size $R$ and diffuseness $a$, the differential cross section scales as (see, e.g., Ref. [8]) $d \sigma / d \Omega \sim\left[J_{1}(q R) / q\right]^{2} \exp (-q a)$, where $q$ $=2 k \sin (\theta / 2)$ is the momentum transfer. Thus, the slope of the cross section in Fig. 1 is due almost entirely to the diffuse character of the ${ }^{4} n$ system. This behavior is universal and would not change with a better description of the potential. In the absence of the Coulomb interaction and other effects, the peak at forward angles yields the total cross section by means of the optical theorem. Its magnitude is due to the overall size of the system. Thus, the only parameter of interest in this case is the diffuseness of the tetraneutron, which determines the backward/forward cross section ratio.

One possible scenario to explain the events seen in Ref. [1] is that the neutrons do not scatter from the ${ }^{4} n$ as a whole, but from a smaller neutron cluster inside the ${ }^{4} n$ system. If the dineutron exists, it might be less diffuse than the ${ }^{4} n$ system. This in consequence could lead to a larger scattering cross section of the protons towards large angles. However, this would also lead to scattered protons with smaller energy. Another possible effect, short-range correlations, which imply large momentum components inside the ${ }^{4} n$ system, are also ruled out as they must be a small component of the total ${ }^{4} n$ wave function. We thus conclude that both of these effects do not help in explaining those events.

One should also expect that additional effects probably imply an even smaller cross section for the ${ }^{4} n$ (or ${ }^{2} n$ )- $p$ elastic scattering at large angles. Since ${ }^{4} n$ is loosely bound there will be a large absorption from the elastic channel due to the inelastic events which disrupt the ${ }^{4} n$ system. The larger the scattering angle, the larger the momentum transfer and the smaller the cross section will be. This effect was not included in our calculations and would pose an even worse scenario for the detection of elastic scattered ${ }^{4} n$ by proton backscattering.

Our calculations suggest that other processes besides elastic scattering are required to sustain the bound $4 n$ interpretation of the events observed in Ref. [1]. A possible case would be the inelastic scattering on protons of very weakly bound systems [9]. However, to produce $\theta_{\text {c.m. }}>100^{\circ}$ recoils, the ${ }^{4} n$ system must interact as a composite object. Given its weak binding, it is unlikely an inelastic interaction could produce a high energy proton.

In conclusion, we have shown that elastic scattering cannot be used to detect a bound ${ }^{4} n$ nucleus [1]. If the ${ }^{4} n$ system exists it has to be very weakly bound and it will also be a very large and diffuse system. It is very unlikely that such a system can scatter protons at angles of $\theta>90^{\circ}$.

We have benefited from useful discussions with N. Orr and F. M. Marqués. This work was supported by the National Science Foundation under Grant Nos. PHY-007091 and PHY-00-70818.
[1] F. M. Marqués et al., Phys. Rev. C 65, 044006 (2002).

[2] N. K. Timofeyuk, J. Phys. G 29, L9 (2003).

[3] C. A. Bertulani and V. Zelevinsky, J. Phys. G 29, 2431 (2003).

[4] S. C. Pieper, Phys. Rev. Lett. 90, 252501 (2003).

[5] G. Bertsch, J. Borysowicz, H. McManus, and G. W. Love, Nucl. Phys. A284, 399 (1977).

[6] W. B. Broste, G. S. Mutchler, J. E. Simmons, R. A. Arndt, and
L. D. Roper, Phys. Rev. C 5, 761 (1972).

[7] S. C. Pieper (private communication).

[8] H. Feshbach, Theoretical Nuclear Physics, Nuclear Reactions (Wiley-Interscience, New York, 1993).

[9] D. Peterson, J. J. Kolata, P. Santi, J. von Schwarzenberg, D. Bazin, and B. M. Sherrill, Phys. Rev. C 67, 014601 (2003). 